\title{
Vierzig Jahre European Federation of Chemical Engineering Europäische Föderation für Chemie-Ingenieur-Wesen
}

\author{
K. Roel Westerterp*
}

\section{Ziele}

Die European Federation of Chemical Engineering EFCE (Europäische Föderation für Chemie-Ingenieur-Wesen) ist ein freiwilliger Zusammenschluß natur- und ingenieur-wissenschaftlicher Verbände Europas. Die Organisation hat sich die wissenschaftliche Weiterentwicklung des Chemieingenieurwesens als Ziel gesetzt und will die Anwendung chemischer Produktionsprozesse fördern. Die Vorstellung der Gründer der EFCE war eine Zusammenarbeit über nationale Grenzen hinaus. Man muß berücksichtigen, daß es in der Zeit um 1950 - ohne Fernsehen, internationalen Tourismus und kurz nach dem zweiten Weltkrieg nur wenige Begegnungen zwischen den europäischen Völkern gab und daß ein gegenseitiges Verständnis und Vertrauen zwischen vielen Ländern nach allem Leiden nur sehr langsam wiedergefunden werden konnte.

Der zweite Weltkrieg hatte einen tiefen Einschnitt hinterlassen und einen Rückgang der Entwicklung in Europa mit sich gebracht. Die USA dagegen waren mit ihrem großen Potential auf der Spitze ihrer industriellen Leistungsfähigkeit. Die zentral gesteuerten Staaten Osteuropas konnten in dieser Zeit ihre Mittel gezielt einsetzen und sie ausschließlich dazu benutzen, sich in großem Maßsstab den Projekten zu widmen, die für sie wichtig waren. Im freicn Europa waren dagegen die materiellen, geistigen und technologisch-wissenschaftlichen Potentiale weit verstreut über kleinere und größere Länder, die alle ihre eigene Geschichte, Kultur und Geisteshaltung hatten.

\section{Gründung der EFCE}

Zwei überzeugte Europäer, der Franzose Dr. Jean Gerard und der Deutsche Dr. Herbert Bretschneider, die schon lange vor dem zweiten Weltkrieg Freunde waren, versammelten 1950 in Mailand eine Gruppe europäischer Chemieingenieure zu ersten Diskussionen um sich. 1952 wurden dann diese Diskussionen auf der Achima in Frankfurt fortgesetzt. Auf dieser Achema hielt Dr. Gerurd einen mitreißenden Plenarvortrag, in dem er sich für eine europäische Zusammenarbeit im Bereich des Chemieingenieurwesens aussprach. Nach dieser ACHEmA führte ein AusschuB von zuerst vier, später dann sechs Europäern die Arbeit weiter. Es

* Prof. Dr. Ir. K. R. Westerterp, C. Eng. FIChemE, University of Twente, Department of Chemical Technology, P.O. Box 217, NL-7500 AE Enschede. Prof. Westerterp war Mitglied des Exekutivausschusses der EFCE von 1969 bis 1992 und Präsident des 1. Weltkongresses für Chemieingenieurwesen in Amsterdam 1976. wurde ein Entwurf für die Satzung eines europäischen Verbandes erarbeitet, der von vielen verschiedenen Standpunkten aus rege diskutiert, überprüft und beleuchtet wurde. Die fertige Satzung wurde dann in viele Länder an die Verbände der Wissenschaftler geschickt, um so deren Meinung und Bewertung zu erfahren.

Am 20. Juni 1953 trafen sich dann in Paris 15 Repräsentanten europäischer Chemieingenieur- und Wissenschaftsgesellschaften aus acht verschiedenen Ländern zum formellen Gründungstreffen der EFCE und fällten dort eine positive Entscheidung für die Zusammenarbeit. Die vereinten Verbände wählten nun die Mitglieder des Ausschusses, der die Föderation führen sollte, das Executive Committee (Exekutivausschuß). Seine ersten Mitglieder waren Dr. Gerard und Dr. Bretschneider, der Engländer Freeth, der Schweizer Dr. Egloff und der Holländer Dr. Vlugter. Weder die Föderation noch der Exekutivausschuß erhielten einen Vorsitzenden. Ein Treffen wurde immer von irgend jemandem aus dem Land, in dem es stattinden sollte, organisiert. Die Wahl dieser Organisationsform bewirkte, daß alle möglichen Tendenzen, den Verband zu dominieren, von vornherein zum Scheitern verurteilt waren. Das General Secretariat (Hauptsekretariat) der EFCE wurde auf Honorarbasis von der Societé Chimique Industrielle in Paris und der Dechema in Frankfurt/M. geführt. Der Exekutivausschuß mußte der General Assembly (Vollversammlung) Rechenschaft ablegen. Diese war mit einem Repräsentanten jedes Mitgliedsverbandes besetzt. Die Vollversammlung nominiert die Mitglieder des Exekutivausschusses, entscheidet über die Satzung usw.

\section{Entwicklungen in den ersten zwanzig Jahren}

Auf dieser Basis konnte sich die EFCE sehr gesund und schnell zu einer international bedeutsamen Institution des Chemieingenieurwesens entwickeln. Die Zusammenarbeit fand überwiegend auf internationalen Treffen, Kongressen und Konferenzen statt. Die persönlichen Kontakte bei diesen Veranstaltungen führten häufig zu gegenseitigen Besuchen in den industriellen und universitären Wissenschaftszentren der europäischen Länder. Es fanden viele neue Entwicklungen statt, von denen nur diejenigen erwähnt werden sollen, die eine größere Wirkung auf den Verband als Ganzes hatten. 1955 wurde eine parallele Organisation gegründet, die European Federation of Corrosion. Korrosion ist von großem Interesse im Chemieingenieurwesen, und man hat schnell erkannt, $\mathrm{da} ß$ die Korrosion ebenso für andere Branchen der Wissenschaft und Industrie wichtig ist. So arbeitete die EFCE gerne bei der Gründung dieser neuen Organisation mit. Dies war ebenso der Fall, als gegen Ende der siebziger Jahre die European Federation of Biotechnology gegründet wurde (1979). 
(2)

O) June 20,1953 the Foundation Assembly of the

\section{EUROPEAN FEDERATION OF CHEMICAL ENGINEERING}

was held in the convention room in the Maison de la Chimie in Paris. It was agreed there by the technical-scientific associations represented by their plenipotentiaries to incorporate the program made by the Working Committee established in Frankfurt (Main) in 1952 on the occasion of the European Meeting of Chemical Engineering as follows and to confirm this resolution by their signatures:

\author{
1. The European Federation of Chemical Engineering was \\ founded on June 20,1953. \\ 2. The regulation submitted to the Foundation Assembly was \\ approved of. \\ 3. The associations having ratified this document confirm beside \\ the acknowledgment of the regulation that they belong to the \\ European Federation of Chemical Engineering.
}

PARIS, JUNE 20,1953

Maison de la Chimie

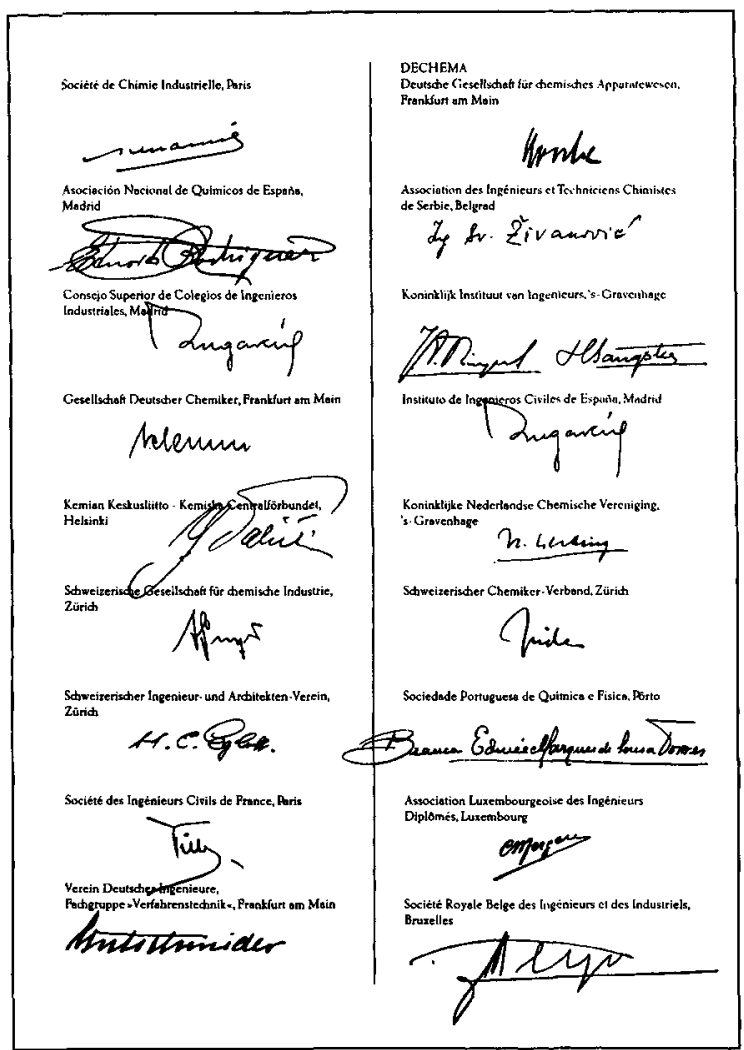

Abb. 1. Die Gründungsurkunde der European Federation of Chemical Engineering.

1955 schloß sich das Indian Institute of Chemical Engineers der EFCE als erste Corresponding Member Society (korrespondierende Mitgliedgesellschaft) an. Das Institut konnte nicht Vollmitglied der EFCE werden, weil es kein europäischer Verband war. Trotzdem bewertete die EFCE das Interesse der Inder so hoch, daß für solche Fälle die Möglichkeit der korrespondierenden Mitgliedschaft geschaffen wurde. Dies führte dazu, daß nach und nach viele Verbände aus der ganzen Welt korrespondierende Mitglieder wurden. Die Verbindung mit den Indern dauerte intensiv über die Jahre fort und führte zu vielen gemeinsamen Konferenzen der europäischen Mitgliedsverbände mit dem Indian Institute. Zur Zeit hat die EFCE dreizehn korrespondierende Mitglieder.

Im Jahre 1956 - seinem Todesjahr - hielt Dr. Jean Gerard eine Jubiläumsrede vor der Society of Chemical Industry in London. Die großen Anstrengungen von Mr. Freeth und der mitreißende Aufruf von Dr. Gerard führten dann im Jahr 1957 zum Eintritt zweier englischer Verbände in die EFCE. Folge war, daß ein drittes Office of the General Secretariat der EFCE in Großbritannien eröffnet wurde.

1957 organisierte die EFCE dann in Amsterdam den ersten Kongreß für chemische Reaktionstechnik, wo vierzehn Vorträge von Experten aus ganz Europa gehalten wurden. Bei diesem Treffen wurde auch die Gründung eines Arbeitskreises für chemische Reaktionstechnik unter dem Dach der EFCE beschlossen. Dies war der erste Fachausschuß der EFCE, dem viele weitere folgten. Ein Fachausschuß hatte sein eigenes Arbeitsgebiet aus dem Bereich des Chemieingenieurwesens und ein eigenes Sekretariat, das von einer Mitgliedsgesellschaft geführt wurde. Jedes Land hatte das Recht, zwei Delegierte für einen Fachausschuß zu nominieren, einen aus der Industrie und einen aus dem universitären Bereich. Die Fachausschüsse organisierten Symposien und Tagungen, studierten und bewerteten die Literatur, beschlossen die Nomenklatur, entwickelten technische Wörterbücher und organisierten ihre eigenen internen wissenschaftlichen Treffen. Den Mitgliedern der Fachausschüsse wurde auch die Aufgabe übertragen, die Informationen, die sie auf den Tagungen erhielten, in ihren Ländern zu veröffentlichen.

Arbeitsk reise wurden zuerst für begrenzte Zeit gegründet. Wenn sie erfolgreich waren, entschied die EFCE, den Arbeitskreis weiterzuführen. Nachdem schon eine ganze Anzahl von Arbeitskreisen gegründet worden war, entwickelte der ExekutivausschuB Ende der sechziger Jahre die Richtlinien für Fachausschüsse. Nachdem mehr und mehr Erfahrungen mit laufenden Arbeitskreisen gewonnen und ihre Leistungen koordiniert worden waren, wurden die Richtlinien noch verbessert. Die Vollversammlung, das Science Advisory Committee (wissenschaftlicher Beratungsausschuß) und der Exekutivausschuß tagten dreimal mit allen Fachausschüssen und ihren Mitgliedern: 1974 in Utrecht, 1981 in Erlangen und 1989 in Aachen.

1964 wurde die Interamerican Confederation of Chemical Engineering gegründet, und 1966 fand in London das erste Treffen zwischen deren Repräsentanten und denen der EFCE statt. Das britische Büro des General Secretariat pflegte die internationalen Kontakte, auch mit der Asian-Pacific Federation of Chemical Engineering, die Ende der siebziger Jahre gegründet worden war.

Bereits 1956 fragte der ExekutivausschuB bei einem AusschuB von Wissenschaftlern für Gutachten an. Dies wurde danach regelmäßig getan, und führte schließlich zur Einrichtung des Science Advisory Council (wissenschaftlicher Beirat) im Jahre 1965. Die Satzungen, die in diesem Jahr verabschiedet wurden, ermöglichten es dem wissenschaftlichen Beratungsausschuß dann, eine wichtige Rolle in der naturwissenschaftlichen Entwicklung Europas zu spielen. Der Ausschuß gründete neue Arbeitsgemeinschaften in Bereichen, in denen Europa zurücklag, und formulierte Probleme, für deren Lösung die Mitgliedsverbände Initiativen ergreifen sollten.

1966 trat der tschechoslowakische Verband als erste wissenschaftliche Organisation Osteuropas der EFCE bei, gefolgt vom polnischen Verband im Jahr 1972. Die jugoslawische Gesellschaft war eines der Gründungsmitglieder der EFCE gewesen. 1971 
wurden dann die ersten Mitglieder osteuropäischer Länder für den Exekutivausschuß und den wissenschaftlichen Beratungsausschuß nominiert. Es war immer die Politik der EFCE gewesen, ihre osteuropäischen Mitglieder so gut wie möglich zu unterstützen, auch wenn diese nicht in der Lage waren, alle Erfordernisse entsprechend der Satzung der EFCE zu erfüllen.

\section{Organisation der EFCE}

Man kann sagen, daß die EFCE in den ersten zwanzig Jahren ihres Bestehens erfolgreich gewachsen ist, sich gut etabliert hat und als eine europäische Körperschaft für Chemieingenieure anerkannt wurde.

Alle bisher hierher diskutierten Entwicklungen führten zu einer Organisationsform, die auch in all den späteren Jahren der Existenz der EFCE ihre Gültigkeit behielt. Sie hat sich als effektiv erwiesen, das Ziel einer intensiven, freiwilligen, europaweiten Zusammenarbeit zwischen den Wissenschaftlern und den Praktikern des Chemieingenieurwesens zu erreichen.

Abb. 2 zeigt das Organisations-Schema der EFCE. Jeder Mitgliedsverband delegiert ein stimmberechtigtes Mitglied in die Vollversammlung (General Assembly) der EFCE. Die korrespondierenden Mitgliedsverbände können Beobachter zur Hauptversammlung schicken, die zwar an den Diskussionen teilnehmen können, aber kein Stimmrecht haben. Die Vollversammlung entscheidet über Anträge, die vom Exekutivausschuß (Executive Committee) oder vom wissenschaftlichen Beirat (Science Advisory Council) eingebracht wurden, zum Beispiel Satzungsänderungen, die Gründung von Fachausschüssen, die Nominierung von Mitgliedern für die Ausschüsse usw. Der Exekutivausschuß hat die Aufsicht über das Management der EFCE und nominiert die individuellen Mitglieder für die Fachausschüsse. Der wissenschaftliche Beratungsausschuß verfolgt die wissenschaftlichen Entwicklungen und leitet die Fachausschüsse. In den Arbeitskreisen hat die wissenschaftliche Arbeit der EFCE ihren Platz, hier werden Tagungen, Workshops und Kongresse organisiert. Die Fachausschüsse unterscheiden sich in Art und Aktivität, aber jeder befaßt sich mit einem bestimmten Bereich des Chemieingenieurwesens, z. B. mit einem Teilgebiet, wie den Trennungsprozessen oder einem Anwendungsgebiet, wie Lebensmittelherstellung oder Meerwasserentsalzung.

1973 zählte die EFCE 48 Mitgliedsgesellschaften und 12 korrespondierende Mitglieder. Der Exekutivausschuß und der wissenschaftliche Beratungsausschuß bestanden jeweils aus neun Mitglie-

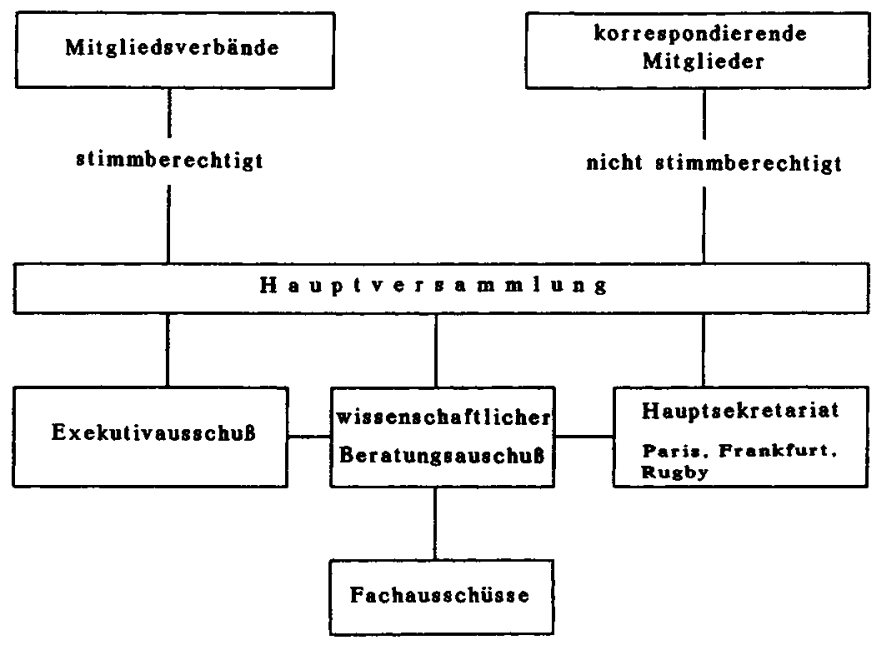

Abb. 2. Organisationsschema der Europäischen Föderation für Chemie-Ingenieur-Wesen. dern, und das Hauptsekretariat wurde von den General-Managern und ihren Mitarbeiterstäben von der DeChEMA in Frankfurt/M. der Institution of Chemical Engineers in London und der Societé de Chimie Industrielle in Paris beauftragt. Es gab insgesamt 11 Fachausschüsse, wovon der älteste der für Chemische Reaktionstechnik war.

In einer Veröffentlichung zur EFCE [1] schlußfolgerte Westerterp im Namen des Exekutivausschusses, daß die Föderation sehr erfolgreich in der Verwirklichung ihrer gesteckten Ziele war und die ursprünglichen Erwartungen der Gründungsmitglieder erfüllt wurden. Eine sehr intensive Zusammenarbeit in allen möglichen Teilbereichen des Chemieingenieurwesens war europaweit erzielt worden. Es waren alle notwendigen Voraussetzungen geschaffen worden, andere Laboratorien, Institute und Fabrikanlagen kennenzulernen, und der gegenseitige Respekt für wissenschaftliche Beiträge wuchs. In der gleichen Publikation wurde auch eine Reihe von Problemen dargelegt und diskutiert:

- die Anzahl und die Kosten der Kongresse. Gab es nicht zu viele Veranstaltungen?

- die Aktivitäten der Fachausschüsse. Publizierten sie verläßlich und in vollem Umfang die neuen Entwicklungen an die Mitglieder ihrer Gesellschaften?

- die interkontinentale Zusammenarbeit. Inwieweit konnte eine Zusammenarbeit auch außerhalb Europas gefördert werden?

- die Ausbildung und die Diplome. Sollte auf eine Vereinheitlichung hingearbeitet werden?

- die Information und Dokumentation. Für das Jahr 1970 ließ sich abschätzen, daß 120 Millionen technische und wissenschaftliche Publikationen erschienen waren, davon 10 bis $15 \%$ im Bereich Chemie und Chemieingenieurwesen.

- die Umweltproblematik. Welche Rolle spielt die EFCE in diesem Bereich?

- die Sicherheit. Auch in diesem Bereich hat die EFCE eine große Verantwortung.

- die Seewasser-Entsalzung. In diesem Gebiet standen große kommerzielle Interessen auf dem Spiel.Wie sollte sich die EFCE als ein wissenschaftlicher Verband ohne kommerzielle Interessen in einer von Konkurrenz bestimmten Welt entscheiden?

Daraus konnte man schließen, $\mathrm{da}$ die kommenden Jahre neue Probleme und Herausforderungen für die EFCE mit sich bringen würden und sie in der Zukunft neue und adäquate Antworten zu finden hätte.

\section{Weitere Entwicklungen in den späteren Jahren}

Wie oben schon erwähnt, liegen viele Probleme auf der Hand, und als eine lebendige Organisation sollte sich die EFCE an deren Lösungen beteiligen. Die EFCE wurde immer stark von außen beeinflußt, was einerseits Probleme andererseits aber auch neue große Möglichkeiten mit sich brachte.

Im September 1972 organisierte der französische Mitgliedsverband ein Symposium mit dem Titel „Chemical Engineering at the Service of Mankind“ in Paris. Die Hälfte der Vortragenden kam aus Übersee. Es war der erste Schritt zu einer internationalen Kooperation. Im Jahr 1976 organisierte der holländische Mitgliedsverband zusammen mit der Interamerican Confederation of Chemical Engineering und dem korrespondierenden japanischen Mitgliedsverband den ersten Weltkongreß für Chemieingenieurwesen. Dieser Kongreß war ein solcher Erfolg, daß zukünftig Weltkongresse im Fünf-Jahres-Zyklus stattfinden sollten. Sie fanden in Toronto und Tokio statt, der vierte WeltkongreB 1991 wurde wieder nach Europa geholt. Er wurde von den deutschen Mitgliedsverbänden, der DeChema und der GVC-VDI in Karlsruhe ausgerichtet. 
Es gab nicht nur eine Tendenz zu immer größeren Zusammenkünften, sondern auch einen Bedarf für lokale Treffen. Spezielle Skandinavien-Konferenzen wurden ins Leben gerufen. Die spanischen Verbände organisierten mehrmals sehr erfolgreich Konferenzen in Barcelona für die Mitgliedsverbände der MittelmeerAnrainerstaaten.

Die Bereiche, in denen sich die Arbeitsgemeinschaften engagieren sollten, waren mehr oder weniger abgesteckt. Es gab aber auch Punkte, die sichtlich von internationaler Bedeutung waren, aber so groß, komplex oder langwierig, daß nicht klar war, in welcher Art und Weise sie zu passenden Projekten für die Fachausschüsse aufbereitet werden konnten. Beispiele hierfür sind die Umweltund Energieproblematik. In solchen Fällen beschloß die Föderation, statt der Arbeitskreise Steering Commitees (Lenkungsausschüsse) einzusetzen, die die Thematik weitblickend bearbeiteten. Kristallisierte sich dabei dann ein Projekt heraus, dessen tiefergehende Bearbeitung lohnend erschien, konnte die EFCE aktiv werden und einen FachausschuB einsetzen oder in einer anderen Weise reagieren.

Der erste Lenkungsausschuß „Aspects of Chemical Engineering in the Protection of the Environment" wurde 1973 eingesetzt. 1974 folgten „Hydrogen Production from Water“ und im Jahr 1978 „Future Changes in the Chemical Industry“. Der erste führte zur Gründung eines Fachausschusses, die beiden anderen zu vielen Berichten, die breit und umfassend diskutiert wurden. Auch bei der Vorbereitung des Weltkongresses 1991 in Karlsruhe wurde eine wichtige Publikation, die „Strategies 2000“, erstellt, und die späteren Ergebnisse des Kongresses wurden danach bewertet und mit diesem politischen Papier verglichen. Die Ausbildung der Chemieingenieure in Europa wurde ebenso intensiv studiert, was zu einer ersten Konferenz mit dieser Thematik im Jahr 1981 in London führte. Die Bedürfnisse der chemischen Industrien in den europäischen Ländern waren jedoch zu unterschiedlich, so daß es noch nicht der richtige Zeitpunkt war, um Vorstellungen über mehr Einheitlichkeit in der universitären Ausbildung zu entwickeln.

Als mit den Jahren die Europäische Gemeinschaft in Brüssel immer bedeutender wurde und speziell immer mehr in die Förderung zur Entwicklung der internationalen Kooperation der Ingenieurwissenschaften einbezogen wurde, hielten es der Exekutivalusschuß und der wissenschaftliche Beratungsausschuß für angebracht, die Kontakte zu den relevanten Ausschußdirektorien zu verbessern. Deshalb wurde das European Community Liaison Committee (Ausschuß für Zusammenarbeit mit der EG) gegründet. Leider war dieser Ausschuß nicht sehr erfolgreich, weil es der EFCE an Möglichkeiten und Organisation fehlte, schnell und tiefgehend auf die Fragen der Beamten der Europäischen Gemeinschaft $z u$ reagieren.

Die drei Kontrollorgane der eher lockeren und informellen EFCE-Organisation hielten ihre Treffen ein- bis zweimal im Jahr ab. Diese vielleicht etwas ungewöhnliche Organisationsform bewährte sich trotzdem sehr gut, was größtenteils auf den personellen Beziehungen, die aufgebaut worden waren, beruhte, und darauf, daß es keine dominierende Gruppe gab. Die endgültige Verantwortung für die wissenschaftliche Arbeit liegt formell bei der Vollversammlung, die tägliche Ausführung beim Exekutivausschuß und beim wissenschaftlichen Beratungsausschuß.

Der wissenschaftliche Teil des Chemieingenieurwesens war überwicgend auf die Arbeitsgemeinschaften delegiert, und die Länder waren für die Organisation und Administration der Arbeitskreise verantwortlich. Die Aktivitäten der Arbeitsgemeinschaften waren überwiegend befriedigend. Die meisten Schwierigkeiten gab es dabei, eine geeignete Sprache beim Dialog mit den Praktikern zu finden, und bei den Finanzen.

Eine Anhäufung von Experten kann zu abgehoben und nach innen gerichtet sein und birgt die Gefahr, daß Diskussionen zum Selbstzweck werden und an den Bedürfnissen der Praktiker vorbeigchen. Nur innerhalb der Ausschüsse der Föderation zu 
arbeiten ist keine gute Voraussetzung, um die praktisch arbeitenden Ingenieure in den Ländern zu erreichen. Eine effektive internationale Zusammenarbeit $\mathrm{mu} ß$ aber gerade dies leisten. Zu diesem Zweck begann der wissenschaftliche Beratungsausschu $B$ in den achtziger Jahren sich in regelmäßigen Abständen mit den Arbeitsgruppen zu treffen und ihren Fortschritt bei Aktivitäten inner- und außerhalb der Föderation zu diskutieren. Die Richtlinien für Fachausschüsse wurden 1978 erneuert, mit dem Passus, daß die Vorsitzenden dem wissenschaftlichen Beratungsausschuß alle drei Jahre einen Rechenschaftsbericht vorzulegen haben und daß dann der wissenschaftliche Beratungsausschuß über die Fortführung oder Auflösung der Arbeitsgruppe zu entscheiden hat. Ebenso wurde eingeführt, daß der Vorsitzende eines Fachausschusses alle drei Jahre neu gewählt werden muß. Er hat außerdem die Pflicht, Protokolle von den Treffen seines Fachausschusses zu erstellen und diese an alle Delegierten zu schicken. Die wiederum haben die Pflicht, diese Informationen an ihre Mitgliedsgesellschaften weiterzugeben, die die jeweiligen Gruppen in ihren Ländern informieren sollen. Andere Probleme gab es mit bestimmten Fachausschüssen, z. B. Konkurrenzkampf mit spezialisierten kommerziellen Organisationen oder manchmal auch untereinander wegen überlappender Aktivitäten oder zeitlich zusammenfallender Konferenzen.

Ein weiterer Schritt wurde mit der Einführung der Individuellen Mitgliedschaft in den Fachausschüssen getan. Dies ermöglichte Personen, an allen Aktivitäten einer Arbeitsgruppe teilzuhaben, jedoch ohne Stimmrecht. Dies war nur den zwei offiziellen Delegierten jedes Landes vorbehalten. Von dieser Vorgehensweise erhoffte man sich, die Effektivität der wissenschaftlichen Arbeit der Fachausschüsse zu verbessern.

Ebenso begann die EFCE, Nachrichten in Briefform herauszugeben. Die erste erschien 1985, die zweite 1987. Heute erscheinen sie regelmäßig. In diesen Nachrichten in Briefform informieren die Mitgliedsgesellschaften über alle Aktivitäten der EFCE, wie Kongresse, Treffen, Revisionen in den Fachausschüssen usw.

1993 nahm ein Ausschuß für Forschungskoordination seine Arbeit auf, mit dem Ziel, die Kooperation zwischen der EFCE und der Europäischen Gemeinschaft zu verbessern. Damit wollte man mehr Einfluß auf zukünftig geplante Forschungsprogramme der Gemeinschaft gewinnen.

Schließlich wurden zwei Gelehrten-Verbände aus der UdSSR Mitglieder der EFCE. Nach der Öffnung der Grenzen Osteuropas änderten auch diese beiden Organisationen ihren Charakter. Die eine war die Abteilung Chemieingenieurwesen der Academy of Science of Russia, die andere die All Union Mendeleev Chemical Society in Rußland. Durch diesen Beitritt wurden viele neue Beitritte aus den früheren kommunistischen Ländern erhalten. Nach vierzig Jahren vereinigt die EFCE nun die Aktivitäten von 63 Mitgliedsverbänden und 23 Fachausschüssen. 13 weitere außereuropäische Gesellschaften sind als korrespondierende Mitglieder angeschlossen.

In dieser Veröffentlichung wird bewußt auf die Erwähnung von Namen verzichtet, mit Ausnahme derer, die bei der Gründung der EFCE mitgewirkt und ihren ersten Exekutivausschuß gebildet haben. Dies wird mit Absicht getan, weil es viele große Wissenschaftler und Persönlichkeiten aus wissenschaftlichen Organisationen gab, die ihr Möglichstes taten, um die Föderation in ihre jetzige Form zu bringen. Sie alle hatten Anteil daran, die wissenschaftliche Zusammenarbeit über die nationalen Grenzen der europäischen Länder hinaus anzuregen und zu fördern. Sie taten dies, weil sie stark an den Nutzen eines geeinten Europa auch im Bereich der Wissenschaft glaubten. Eine Ausnahme soll von den eben beschriebenen Prinzipien gemacht werden: Mehr als dreißig Jahre leitete Prof. Dr. Dieter Behrens das Büro des Hauptsekretariats in Frankfurt/M. Als ein überzeugter Europäer leistete er einen großen Anteil an allen EFCE-Aktivitäten in diesen Jahren. Er starb ein halbes Jahr nach seiner Pensionierung im Juni 1992, und die EFCE trauert sehr um den Verlust einer ihrer wohl faszinierendsten Persönlichkeiten. Prof. Behrens war vor allem ein überzeugter Europäer, der es verstand, die Gemeinschaft der Chemieingenieure rund um die Welt zu einigen. Dank seiner großen diplomatischen Fähigkeiten konnte er die Menschen aus Wissenschaft und Industrie zusammenführen.

\section{Quo vadis EFCE?}

In den vierzig Jahren des Bestehens der EFCE haben große Veränderungen in Europa stattgefunden. Die politische Teilung Europas in zentralistisch regierte Staaten und in solche mit freier Marktwirtschaft ist jetzt vorüber. Dies eröffnet nun alle Möglichkeiten für einen freien Meinungsaustausch und eine Zusammenarbeit zwischen allen Wissenschaftlern Europas ohne Restriktionen, soweit es die finanziellen Mittel erlauben. Gefordert ist nun eine intensive Zusammenarbeit zwischen den Wissenschaftlern der früheren beiden „Blöcke“; weil jeder für sich lange unabhängig wissenschaftlich gearbeitet hat, kann nun einer vom anderen im Austausch vieles lernen.

Weiterhin wächst im westlichen Teil Europas immer mehr der Einfluß der Europäischen Gemeinschaft. Sie hat eine immer bedeutender werdende Rolle für die Förderung des wissenschaftlichen Fortschritts und die Zusammenarbeit. In Zukunft sollen auch die osteuropäischen Staaten an dieser Gemeinschaft teilhaben. Dies aber erfordert Aktivitäten auf einer "supranationalen“ Ebene und eine einflußreichere Rolle der EFCE. In ihren eigenen Staaten unterstützen viele Mitgliedsgesellschaften die nationalen Interessen ihrer Parlamente in für die Föderation relevanten Bereichen, wie Forschungspolitik, Sicherheit, Gesundheit, Energie und Umwelt. Die Föderation sollte sich als der wichtigste Gegenpart für das europäische Chemieingenieurwesen dafür einsetzen, die Gemeinschaft zu unterstützen und bei der Lösung ihrer Probleme mitzuwirken.

Diese beiden großen Entwicklungslinien hin zu einem geeinten Europa erfordern auch organisatorische Änderungen in der EFCE. Sie muß eine Rolle einnehmen, die es ihr als eine Organisation von Chemieingenieuren ermöglicht, bei der Identifizierung der Bedürfnisse und Möglichkeiten in der Forschung des Chemieingenieurwesens mitzuwirken, so z. B. durch die Erstellung von Fachpublikationen zu brisanten aktuellen und die Zukunft betreffenden Themen, so daß die EFCE zu einem wichtigen Ratgeber für die Europäische Gemeinschaft wird.

Deshalb wurden auf der Vollversammlung 1993 in Prag Vorschläge für eine grundlegende Änderung der Organisation der EFCE diskutiert. Die Wahl eines EFCE-Präsidenten für drei Jahre ist der erste Schritt. Es wird seine Pflicht sein, zusammen mit dem Exekutivausschuß weitere zukünftige Änderungen anzuregen, abzuschätzen und vorzubereiten. Wir befinden uns auf der Schwelle einer neuen Ära für die EFCE mit großen Herausforderungen, hoffnungsvollen Errungenschaften und Beiträgen für die Zukunft im geeinten Europa. Dazu wünschen wir Prof. Hanns Hofmann als erstem EFCE-Präsidenten viel Erfolg.

Eingegangen am 30. September 1993 [B 5799]

\section{Literatur}

[1] Westerterp, K. R.: Europäische Föderation für ChemieIngenieur-Wesen. Ergebnisse und Probleme, Achema-Jahrbuch 1971/1973, Band I, S. 6/10. 